\title{
Epidemiological, clinical, outcome and antibiotic susceptibility differences between PVL positive and PVL negative Staphylococcus aureus infections in Western Australia: a case control study
}

Peter Boan ${ }^{1 *}$, Hui-Leen Tan ${ }^{1,2}$, Julie Pearson ${ }^{1,2}$, Geoffrey Coombs ${ }^{1,2}$, Christopher H Heath ${ }^{1}$ and James Owen Robinson ${ }^{1,2}$

\begin{abstract}
Background: Panton Valentine Leukocidin (PVL) has been associated with invasive Staphylococcus aureus soft tissue and pneumonic infections.

Methods: From September 2007 to January 2009 at Royal Perth Hospital we tested for the PVL gene in S. aureus isolates from an invasive site, a suspected PVL-related soft tissue infection and all MRSA isolates. We could access medical records for $141 \mathrm{PVL}$ positive (PVL + ve) infections and compared these to a control group comprised of 148 PVL negative (PVL-ve) infections.

Results: In the PVL + ve group 62 isolates were MRSA (48 were ST93-MRSA-IV) and 79 isolates were methicillin-sensitive S. aureus, and in the PVL-ve group 56 were MRSA (50 were WA-MRSA strains) and 92 were methicillin-sensitive S. aureus. We found the presence of PVL to be significantly associated with younger age, aboriginality, intravenous drug use, community acquisition, shorter length of hospital stay and lower mortality at 1 year. Overall PVL + ve infections more often required surgical intervention $(73.0 \%$ versus $44.6 \%, p<0.001)$ and were less often polymicrobial $(8.5 \%$ versus $41.2 \%$, $p<0.001) . P V L+$ ve isolates were more often susceptible to clindamycin ( $87.9 \%$ versus $73.0 \%, p=0.002)$.

Conclusions: This study demonstrates that PVL + ve infections are associated with a distinct clinical picture, predominantly pyogenic skin and soft tissue infections often requiring surgery, disproportionately affecting patients who are younger, indigenous or with fewer health-care risk factors.
\end{abstract}

Keywords: Staphylococcus aureus, MRSA, Panton Valentine Leucocidin, PVL

\section{Background}

Staphylococcus aureus is a common human pathogen which causes a wide variety of infections. Over the past decades, S. aureus epidemiology has dramatically changed with the appearance of methicillin resistant strains in the community (CA-MRSA), when such strains had previously only been described in hospitals. Since its ini-

\footnotetext{
* Correspondence: Peter.Boan@health.wa.gov.au

'Department of Microbiology and Infectious Diseases and PathWest Laboratory Medicine WA, Royal Perth Hospital, Wellington St, Perth, WA 6000, Australia

Full list of author information is available at the end of the article
}

tial description [1], CA-MRSA has emerged throughout the world accounting for nearly $70 \%$ of S. aureus recovered from patients with skin and soft tissue infections attending an emergency department in the United States [2]. Although diverse clones are responsible for the emergence of CA-MRSA world-wide, the major global clones harbour the Panton-Valentine leukocidin (PVL) [3], a pore-forming toxin that disrupts host cell membranes and which is associated with recurrent boils [4], necrotising fasciitis [5] and necrotising pneumonia [6]; 
though is infrequently associated with asymptomatic carriage [7]. Naturally PVL has been well studied in CA-MRSA though our study contributes also to the expanding data on PVL in methicillin sensitive $S$. aureus (MSSA), and describes aspects of local Western Australian S. aureus epidemiology, clinical outcomes, antimicrobial susceptibility and distribution of MRSA types related to PVL.

\section{Methods}

\section{Study design}

Royal Perth Hospital is an adult tertiary centre with a 955-bed capacity. The Australian Collaborating Centre for Enterococcus and Staphylococcus Species (ACCESS) Typing and Research performs PVL testing on (a) all $S$. aureus grown from sites of invasive disease (e.g. tissue, bone/joint, blood), (b) S. aureus from some skin/soft tissue specimens (where there is some suggestion of aggressive infection such as abscess formation, determined by the microbiology scientist's interrogation of the clinical notes and specimen description on the laboratory request form), (c) all MRSA isolates including nasal and skin colonisation swabs. For this study we excluded $S$. aureus colonisation, defined as $S$. aureus growth without need for surgery or antimicrobial therapy, and duplicate isolates from the same patient were not included. We collated data from all $170 \mathrm{PVL}+\mathrm{ve}$ isolates between September 2007 and January 2009. Medical records were unavailable for 7 cases and $22(13.5 \%)$ colonising isolates were excluded, leaving $141 \mathrm{PVL}+$ ve infections during the 17 month period. Of 1,146 PVL-ve isolates during the time period, 836 were MRSA cultured from nasal or skin colonisation swabs (72.9\%). From the remaining 310 cases to approximate numbers in the PVL + ve group, 148 PVL-ve $S$. aureus infections were selected as "controls" by selecting every second isolate chronologically.

Demographics and co-morbidity data were collected from the medical record, and all cause mortality at 30days and 1-year was determined from clinical information systems data-linked to the Western Australian Registry of Deaths. We recorded whether empirical antibiotic choice was effective (defined if the isolate was susceptible in vitro to any of the antibiotics prescribed), empirical use of protein synthesis inhibitors (lincosamide/linezolid), and lincosamide/linezolid use within 3 days of isolate acquisition. Infection was defined as "hospital acquired" if the isolate was collected more than 48 hours after admission and symptoms were not present on admission. "Healthcare associated" infection occurred in the community in the presence of at least one of the following three risk factors: (a) in the past 12 months a history of surgery, hospitalisation, dialysis or residence in a long term care facility, (b) presence of an invasive device at the time of infection onset, (c) a history of MRSA infection or colonisation. "Community acquired" infection occurred in the community without any of the above risk factors [8]. The study was approved by the Royal Perth Hospital Ethics Committee. Patient informed consent was waived in accord with guidelines of the National Health and Medical Research Council of Australia for a clinical audit [9].

\section{Microbiological methods}

S. aureus was identified using routine laboratory methods (catalase-positive, tube coagulase-positive Gram-positive cocci). Antibiotic susceptibilities were determined by disk diffusion using CLSI methodology and breakpoints [10]. Methicillin resistance was determined by demonstrating cefoxitin resistance. Genes encoding PVL were detected with the nuclease $(n u c)$ and $m e c A$ genes by an in-house real time polymerase chain reaction (PCR) assay using the Light Cycler 2.0 (Roche Diagnostics) as previously described [11]. Briefly a single colony incubating for less than 48 hours was first treated with lysostaphin, proteinase $\mathrm{K}$ and tris buffer. Cell lysate was added to the in-house PCR master mix containing $1 \times$ LightCycler Master Hybridization Probes mixture (Roche Molecular Biochemicals), $5 \mathrm{mM} \mathrm{MgCl} 2,0.5 \mu \mathrm{M}$ forward and reverse primers and two FRET (Fluorescence Resonance Energy Transfer) probes $(0.2 \mu \mathrm{M})$ each for PVL, mec and nuc genes. Two microlitres of sample was added to 18 microlitres of mastermix. We performed 45 cycles of PCR with 3 channel detection of the gene products followed by melt curve analysis. Six controls were used for each PCR run: MRSA PVL negative, MSSA PVL positive, MR coagulase negative Staphylococcus, MS coagulase negative Staphylococcus, a mutant MRSA PVL negative strain (which demonstrates low efficiency $n u c$ PCR), and a negative control. A positive result for $P V L$, mec or $n u c$ required exponential increase in fluorescence within 40 PCR cycles and a characteristic melting temperature. All MRSA and no MSSA isolates were typed by antibiogram, Christensen's urea broth, and previously described coagulase gene PCR-Restriction Fragment Length Polymorphisms assay and contour clamped Homogenous Electric Field Electrophoresis [12]. Multi Locus Sequence Typing and Staphylococcal Chromosomal Cassette mec typing by PCR were performed on some isolates according to prior description [13].

\section{Statistical methods}

Data was examined in IBM SPSS Statistics version 19 (SPSS Inc., Chicago, IL, USA). Continuous variables were examined by the t-test or Mann-Whitney U-test as appropriate and categorical variables by the Chi-Squared or Fisher's exact test as appropriate. Logistic regression 
was performed entering all variables non-sequentially. For all statistical analysis a p-value $<0.05$ was considered to be significant.

\section{Results}

Patients with PVL + ve S. aureus infections compared to the control group were younger (median age 37 vs 58 years, $\mathrm{p}<0.001)$, more often male $(72.3 \%$ vs $62.8 \%, \mathrm{p}=$ $0.085)$, more commonly indigenous $(34.0 \%$ versus $12.2 \%$, $\mathrm{p}<0.001)$, more often intravenous drug users $(16.3 \%$ vs $6.8 \%$ respectively, $\mathrm{p}=0.011$ ), but were less likely to have haematological malignancy $(0.0 \%$ vs $4.1 \%, \mathrm{p}=0.016)$ or a solid tumour $(1.4 \%$ vs $9.5 \%, \mathrm{p}=0.003)$. Long-term care facility residents were rarely infected with $\mathrm{PVL}+\mathrm{ve}$ strains, being $1.4 \%$ of PVL + ve cases compared to $14.9 \%$ of controls $(\mathrm{p}<0.001)$. The majority of PVL + ve infections were community acquired in contrast to the control group which were often healthcare associated or hospital acquired infections (Table 1).

Skin and soft tissue was the most common site of infection in both groups, being significantly more common in the PVL + ve compared to the PVL-ve group (81.6\% vs $63.5 \%, \mathrm{p}<0.001$ ), and abscesses accounted for a larger percentage of these infections in the $\mathrm{PVL}+$ ve group versus controls $(87.0 \%$ compared to $41.5 \%$ respectively, $\mathrm{p}<0.001)$. Bacteraemia occurred in 50 cases, of which $13(26.0 \%)$ were PVL + ve $(\mathrm{p}<0.001)$. Bone and joint infections were the third most common site of infection being less common in the PVL + ve than the control group $(3.5 \%$ vs $8.8 \%$ respectively, $\mathrm{p}=0.066)$. Seven

Table 1 Epidemiological associations with PVL status

\begin{tabular}{|c|c|c|c|}
\hline Characteristic & $\begin{array}{l}\text { PVL positive } \\
(n=141)\end{array}$ & $\begin{array}{l}\text { PVL negative } \\
(n=148)\end{array}$ & p-value \\
\hline Age (median) & 37 & 58 & $<0.001$ \\
\hline Male & $102(72.3)$ & $93(62.8)$ & 0.085 \\
\hline Indigenous & $48(34.0)$ & $18(12.2)$ & $<0.001$ \\
\hline Intravenous Drug Use & $23(16.3)$ & $10(6.8)$ & 0.011 \\
\hline Haematological malignancy & $0(0)$ & $6(4.1)$ & 0.016 \\
\hline Solid malignancy & $2(1.4)$ & $14(9.5)$ & 0.003 \\
\hline Diabetes Mellitus & $31(22)$ & $44(29.7)$ & 0.133 \\
\hline Immunosuppressive treatment & $7(5)$ & $14(9.5)$ & 0.141 \\
\hline HIV positive & $3(2.1)$ & $2(1.4)$ & 0.613 \\
\hline Pre-existing skin condition & $4(2.8)$ & $4(2.7)$ & 0.945 \\
\hline Hospitalisation in the past year & $50(35.5)$ & $92(61.2)$ & $<0.001$ \\
\hline Long term care facility resident & $2(1.4)$ & $22(14.9)$ & $<0.001$ \\
\hline $\begin{array}{l}\text { "Community acquired" } \\
\text { infection }\end{array}$ & $87(61.7)$ & 35 (23.6) & $<0.001$ \\
\hline $\begin{array}{l}\text { "Healthcare associated" } \\
\text { infection }\end{array}$ & 46 (32.6) & $78(52.7)$ & $<0.001$ \\
\hline "Hospital acquired" infection & $8(5.7)$ & $35(23.6)$ & $<0.001$ \\
\hline
\end{tabular}

Number (\%)
(5.0\%) lung infections were due to PVL + ve S. aureus (4 empyema, 1 lung abscess, 2 pneumonia) compared to $3(2.0 \%)$ due to PVL-ve S. aureus (2 pneumonia and one empyema, $\mathrm{p}=0.172)$. For $12(8.5 \%) \mathrm{PVL}+$ ve infections another pathogen was grown from the same specimen compared to $61(41.2 \%)$ of the control group. This difference was most marked for skin and soft tissue infections though a trend was also seen in bloodstream and bone/joint samples (Table 2).

Antimicrobial susceptibility results are shown in Table 3. Of note, $87.9 \%$ of PVL + ve isolates tested susceptible (includes a negative $\mathrm{D}$-test) to clindamycin compared with $71.6 \%$ of the control isolates $(\mathrm{p}=0.002)$. Erythromycin and mupirocin susceptibility rates were also higher for PVL + ve strains compared to controls. Overall, only $80.1 \%$ of MRSA isolates were susceptible to clindamycin (data not shown).

Queensland clone (ST 93-IV, $\mathrm{n}=48$ ) was the predominant PVL + ve MRSA strain, with smaller numbers of Western Samoan Phage Pattern strains (ST30-IV, $\mathrm{n}=5$ ), USA 300 (ST8- IV, n=4), WA1-MRSA strains (ST1-IV, $\mathrm{n}=3$ ), Taiwan strain (ST59-VT, $\mathrm{n}=1)$ and EMRSA-15 (ST22-IV, $\mathrm{n}=1$ ). For PVL-ve MRSA isolates, most were WA-MRSA strains (WA-MRSA-1 [ST1- IV], WAMRSA-2 [ST78-IV], WA-MRSA-3 [ST5-IV], WA-MRSA75 [ST45-IV], $\mathrm{n}=50$ ) with few overseas strains: EMRSA-15 (ST22-IV, $\mathrm{n}=6$ ). No MSSA isolates were typed.

Empirical antimicrobial therapy was effective in 75 (53.2\%) PVL + ve cases and 90 (60.8\%) control cases $(p=0.191)$. Cases not receiving effective empiric therapy were predominantly because of unexpected methicillin resistance (103 cases, 83.1\%, OR 9.1 [4.7-10.7 95\% CI]) or as infections were polymicrobial (39 cases, $31.5 \%$, OR 1.3 [0.99-1.70 95\% CI]). Lincosamides or linezolid

Table 2 Specimen type by PVL status

\begin{tabular}{lllll}
\hline Specimen type & Subcategory & $\begin{array}{l}\text { PVL positive } \\
(\mathbf{n}=\mathbf{1 4 1})\end{array}$ & $\begin{array}{l}\text { PVL negative } \\
(\mathbf{n}=\mathbf{1 4 8})\end{array}$ & p-value \\
\hline All & Polymicrobial & $12(8.5)$ & $61(41.2)$ & $<0.001$ \\
Skin/soft tissue & Total & $115(81.6)$ & $94(63.5)$ & $<0.001$ \\
& Polymicrobial & $10(8.7)$ & $51(54.3)$ & $<0.001$ \\
(a) Cellulitis/ & Total & $15(13.0)$ & $55(58.3)$ & $<0.001$ \\
wound swab & Polymicrobial & $5(33.3)$ & $33(60.0)$ & 0.006 \\
(b) Abscess & Total & $100(87.0)$ & $39(41.5)$ & $<0.001$ \\
soft tissue & Polymicrobial & $5(5.0)$ & $18(46.2)$ & $<0.001$ \\
Blood & Total & $13(9.2)$ & $37(25.0)$ & $<0.001$ \\
& Polymicrobial & $0(0.0)$ & $3(8.1)$ & 0.290 \\
Bone/Joint & Total & $5(3.5)$ & $13(8.8)$ & 0.066 \\
& Polymicrobial & $0(0.0)$ & $5(38.5)$ & 0.103 \\
Lung & Total & $7(5.0)$ & $3(2.0)$ & 0.172 \\
Renal & Total & $0(0.0)$ & $1(0.7)$ & 0.328 \\
\hline Number (\%). & & & &
\end{tabular}


Table 3 Antibiotic susceptibility by PVL status

\begin{tabular}{llll}
\hline Antibiotic & $\begin{array}{l}\text { PVL positive } \\
(\mathbf{n = 1 4 1 )}\end{array}$ & $\begin{array}{l}\text { PVL negative } \\
(\mathbf{n = 1 4 8})\end{array}$ & p-value \\
\hline Methicillin & $79(56.0)$ & $92(62.2)$ & 0.289 \\
Clindamycin & $124(87.9)$ & $106(71.6)$ & 0.002 \\
Erythromycin & $122(86.5)$ & $105(70.9)$ & 0.005 \\
Mupirocin & $139(98.6)$ & $137(92.6)$ & 0.040 \\
\hline
\end{tabular}

Number (\%).

(protein synthesis inhibitors) were more frequently added to therapy and surgery was more often required (73.0\% versus $44.6 \%, \mathrm{p}<0.001)$ for $\mathrm{PVL}+$ ve infections compared to controls (Table 4).

Rates of admission to high dependency unit and/or intensive care unit were lower $(5.7 \%$ vs $11.5 \%, \mathrm{p}=0.079)$ and length of hospital stay was significantly shorter (median 5.0 vs 13.5 days, $\mathrm{p}<0.001$ ) for $\mathrm{PVL}+$ ve infections compared to controls. Both 30 day and 1 year all cause mortality were lower with PVL + ve infections than for PVL-ve infections $(2.8 \%$ vs $6.8 \%, \mathrm{p}=0.121 ; 6.4 \%$ vs $19.6 \%, \mathrm{p}=0.001$ ) (Table 4). The 30-day mortality was not influenced by adjunctive protein synthesis inhibitor therapy $(2.7 \%$ vs $5.3 \%$ for PVL + ve infections and controls, respectively; $\mathrm{p}=0.534$ ). Interestingly, outcome was not influenced by initiation of effective empiric therapy (data not shown).

We performed a logistic regression analysis of PVL status incorporating variables aboriginality, age, intravenous drug use (IVDU), community acquisition, abscess, surgery and hospital length of stay. Statistically significant independent associations with PVL were found for aboriginality (OR 3.89, 1.83-8.25 95\% CI, $\mathrm{p}<0.001$ ), age (OR 0.96, 0.94-0.98 95\% CI, $\mathrm{p}<0.001$ ), community acquisition (OR 2.47, 1.32-4.62 95\% CI, $\mathrm{p}=0.005$ ), abscess (OR 3.45, 1.78-6.69 95\% CI, p < 0.001 ), but were not found for IVDU (OR 1.67, 0.64-

Table 4 Outcomes by PVL status

\begin{tabular}{llll}
\hline Management or Outcome & $\begin{array}{l}\text { PVL positive } \\
(\mathbf{n = 1 4 1 )}\end{array}$ & $\begin{array}{l}\text { PVL negative } \\
(\mathbf{n}=\mathbf{1 4 8})\end{array}$ & p-value \\
\hline Initial antibiotics effective & $75(53.2)$ & $90(60.8)$ & 0.191 \\
Lincosamide/linezolid initial Tx $^{\mathrm{a}}$ & $9(6.4)$ & $4(2.8)$ & 0.141 \\
Lincosamide/linezolid 3d $^{\mathrm{b}}$ & $29(20.8)$ & $10(6.8)$ & $<0.001$ \\
Surgery performed & $103(73.0)$ & $66(44.6)$ & $<0.001$ \\
HDA/ICU admission & $8(5.7)$ & $17(11.5)$ & 0.079 \\
Median length of stay-days & $5.0(0-81)$ & $13.5(0-638)$ & $<0.001$ \\
(range) & & & \\
Death within 30 days & $4(2.8)$ & $10(6.8)$ & 0.121 \\
Death within 1 year & $9(6.4)$ & $29(19.6)$ & 0.001
\end{tabular}

${ }^{\mathrm{a}}$ Lincosamide or linezolid prescribed in the initial therapy.

bincosamide or linezolid added to therapy within 3 days of isolate collection. ${ }^{\mathrm{C}}$ High dependency area or intensive care unit admission.

Number (\%).
$4.3795 \% \mathrm{CI}, \mathrm{p}=0.29$ ), surgery (OR $1.77,0.92-3.41$ $95 \% \mathrm{CI}, \mathrm{p}=0.089$ ), or hospital length of stay (OR 0.98 , 0.97-1.00 95\% CI, $\mathrm{p}=0.100$ ).

For the subset of MRSA isolates, PVL was significantly associated with younger age (median 37 vs 50 years, $\mathrm{p}<$ $0.001)$, IVDU ( $p=0.047)$, abscess formation $(\mathrm{p}<0.001)$, community acquisition $(\mathrm{p}<0.001)$, monomicrobial infection $(\mathrm{p}<0.001)$, surgery $(\mathrm{p}=0.001)$, shorter length of stay (median 3 vs 10 days, $\mathrm{p}<0.001$ ), but not significantly associated with aboriginality $(\mathrm{p}=0.091)$.

For the subset of invasive isolates, PVL was significantly associated with aboriginality $(\mathrm{p}=0.001)$, but not significantly associated with age (median 41 vs 45 years, $\mathrm{p}=0.378)$, community acquisition $(\mathrm{p}=0.171)$, IVDU $(p=1.000)$, surgery $(p=0.433)$, or length of stay (median 5 vs 7 days, $\mathrm{p}=0.300$ ).

\section{Discussion}

We found PVL + ve S. aureus less frequently associated with colonisation than controls $(13.5 \%$ vs at least $72.9 \%$, respectively). A Polish group has replicated these findings, showing that $85.0 \%$ of $S$. aureus isolates causing furunculosis were $\mathrm{PVL}+\mathrm{ve}$, compared to asymptomatic blood donors where less than $1.0 \%$ of nasal swabs yielded positive strains [14]. Likewise, the prevalence of PVL + ve S. aureus colonisation was $4.5 \%$ in indigenous and rural Malaysian communities [15]. A north-east Australian study of nasal colonisation of volunteers and patients seeing their general practitioner with a non-infectious illness (total sample size 699) showed that only $1.0 \%(2 / 202)$ of $S$. aureus isolates recovered were PVL + ve, in contrast to results showing $55.0 \%$ of non-multiresistant MRSA and $13.0 \%$ of MSSA clinical isolates in this region were PVL + ve [16]. Conversely, a New Zealand study of clinical isolates versus asymptomatic nasal isolates of methicillin sensitive $S$. aureus found the prevalence of PVL + ve strains was no different between these groups $(37.0 \%$ vs $31.0 \%, p=0.33)$ [17]. Heterogeneity between such studies may be due to differing ability of various PVL + ve clones to cause colonisation.

Our study shows strong associations between PVL + ve $S$. aureus infections and younger age, male gender, aboriginality, intravenous drug use and community acquired infection compared to healthcare-associated infection. Conversely for PVL-ve infections, healthcare-associated epidemiological factors such as malignancy or nursing home residency were significantly more common. These associations were true for MRSA and MSSA. Our findings support other recent reports that include MSSA: A study from the Northern Territory of Australia of 223 PVL + ve and 255 PVL-ve S. aureus isolates (methicillin susceptible and resistant) showed that presence of PVL was associated with younger age, fewer co-morbidities 
and community onset [18]. Likewise, the aforementioned Auckland study of 124 PVL + ve and 211 PVL-ve MSSA isolates found PVL associated with younger age, Pacific ethnicity, surgical drainage needed and community onset [17].

One hypothesis for the higher rates of PVL + ve $S$. aureus infections amongst younger patients is that older patients through previous exposure might have developed immunity to this toxin. Indeed, in the 1950s, a phage 80/81 strain, now MLST ST30, became pandemic causing outbreaks of skin and soft tissue infections and pneumonia, especially in children [19]. A leukotoxin was thought responsible for the virulence and transmissibility of the strain. One postulate suggests this epidemic had waned by the mid 1960s due to the advent of penicillinase-resistant penicillins, but potentially the epidemic waned from rising immunity to PVL within the general population; supporting this concept it was shown the toxic effect of PVL on cells could be neutralised by immunoglobulins from patients convalescing from infections caused by this strain [20]. Furthermore, Gauduchon et al. have reported increasing prevalence of antibodies to PVL with increased age, further supporting an immunity hypothesis [21].

Our study shows that PVL + ve S. aureus is strongly associated with skin and soft tissue infection, predominantly abscesses. On the contrary, there is a suggestion that PVL + ve $S$. aureus infrequently caused bacteraemia or bone and joint infection. These findings have consistently been reported in the literature $[2,4,18,22,23]$ summarised in the meta-analysis by Shallcross et al. [24], and together with PVL + ve $S$. aureus strains tending to affect younger patients with less co-morbidities, partly explains the overall lower mortality from PVL + ve $S$. aureus disease [17]. However, PVL + ve S. aureus strains more often lead to relapse of infection [14] and can cause epidemics of skin/soft tissue infections as reported by rising Emergency Department attendances for furunculosis, creating significant disease burden for healthcare systems [25]. Whilst PVL + ve S. aureus strains generally cause a relatively benign disease spectrum, life threatening infections such as necrotising pneumonia are well documented $[6,26]$. This particular manifestation of PVL + ve $S$. aureus disease is rare, presumably because additional co-factors are needed to mediate this severe clinical picture, for example preceding viral infection [6]. Unfortunately, our sample size was too small to substantiate any difference between PVL + ve and PVL-ve $S$. aureus lung infections.

We believe our study failed to demonstrate any benefit of adding a protein synthesis inhibitor (e.g. lincosamide or linezolid) to therapy of PVL + ve S. aureus infections because surgical drainage of abscesses, the commonest presentation of these infections, is the mainstay of their treatment with antibiotics playing only a limited role in treatment outcomes. Nonetheless, addition of a protein synthesis inhibitor has been recommended for severe invasive PVL + ve S.aureus lung disease [27].

The most significant limitation of this study is the potential for selection bias. Crucially we have selected skin/ soft tissue $S$. aureus isolates for PVL testing because we expected to find the PVL gene with a history of abscess formation, based on previous literature. This finding is borne out in the association of PVL and abscess formation in the study, however there is a risk we have biased the PVL + ve group with abscess specimens falsely elevating the percentage of skin/soft tissue to invasive infections, potentially confounding bacteraemia rates or outcome data as examples. A multivariate analysis including abscess as a variable and subset analysis of isolates sent for PVL testing for different reasons (MRSA or invasive isolates) are attempts to account for this selection bias, and many variables associated with PVL in the primary analysis continued to show significant associations in these analyses. Ultimately testing all $S$. aureus laboratory isolates for the PVL gene would have been more ideal. Due to the dominance of ST 93-IV in the PVL + ve MRSA group, it is possible other factors associated with this clone influenced outcomes in this group. Additionally, lack of MSSA typing leads to an incomplete understanding of complete clonal makeup of either group. Other limitations of the study include: the study was performed in an adult teaching hospital in Perth, Western Australia and might not necessarily represent the clinical and molecular epidemiology of invasive $S$. aureus infection in other jurisdictions in Australia or in the paediatric or Aboriginal populations; the study was non-interventional and may lack statistical power to detect differences for some outcomes; clinical data relied on hospital medical records rather than a specific case report form and therefore we could have underestimated the frequency of risk factors and additional data points.

\section{Conclusions}

In summary, our study demonstrates that PVL + ve MRSA and MSSA are associated with a specific clinical picture, predominantly pyogenic skin and soft tissue infections which are often monomicrobial and require surgery, more often involving patients who are younger, indigenous or with fewer healthcare risk factors. Whether immunisation or new therapeutic strategies can overcome this worldwide epidemic remains to be seen.

\section{Abbreviations}

CA-MRSA: Community acquired methicillin resistant Staphylococcus aureus; CLSI: Clinical Laboratory Standards Institute; IVDU: Intravenous drug use; MRSA: Methicillin resistant Staphylococcus aureus; MSSA: Methicillin sensitive Staphylococcus aureus; PCR: Polymerase chain reaction; PVL: Panton Valentine Leucocidin; ST: Sequence type; WA: Western Australia. 


\section{Competing interests}

The authors declare that they have no competing interests.

\section{Authors' contributions}

Design of study: PB, JOR. Acquisition of data: PB, JOR, HLT, JP, GC. Analysis of data: PB, JOR, GC, CH. Drafting of manuscript: PB, JOR. Revision of manuscript: $\mathrm{PB}, \mathrm{JOR}, \mathrm{CH}, \mathrm{GC}, \mathrm{HLT}$, JP. All authors read and approved the final manuscript.

\section{Author details}

${ }^{1}$ Department of Microbiology and Infectious Diseases and PathWest Laboratory Medicine WA, Royal Perth Hospital, Wellington St, Perth, WA 6000, Australia. ${ }^{2}$ Australian Collaborating Centre for Enterococcus and Staphylococcus Species (ACCESS) Typing and Research, PathWest Laboratory Medicine WA and Curtin University of Technology, GPO Box X2213, Perth, WA 6847, Australia.

\section{Received: 17 April 2014 Accepted: 23 December 2014}

Published online: 09 January 2015

\section{References}

1. Udo EE, Pearman JW, Grubb WB. Genetic analysis of community isolates of methicillin-resistant Staphylococcus aureus in Western Australia. J Hosp Infect. 1993:25:97-108.

2. Moran GJ, Krishnadasan A, Gorwitz RJ, Fosheim GE, McDougal LK, Carey RB, et al. Methicillin-resistant S. aureus infections among patients in the emergency department. N Engl J Med. 2006;355:666-74.

3. Vandenesch F, Naimi T, Enright MC, Lina G, Nimmo GR, Heffernan H, et al. Community-acquired methicillin-resistant Staphylococcus aureus carrying Panton-Valentine leukocidin genes: worldwide emergence. Emerg Infect Dis. 2003;9:978-84

4. Wehrhahn MC, Robinson JO, Pearson JC, O'Brien FG, Tan HL, Coombs GW, et al. Clinical and laboratory features of invasive community-onset methicillin-resistant Staphylococcus aureus infection: a prospective case-control study. Eur J Clin Microbiol Infect Dis. 2010:29:1025-33.

5. Miller LG, Perdreau-Remington F, Rieg G, Mehdi S, Perlroth J, Bayer AS, et al. Necrotizing fasciitis caused by community-associated methicillin-resistant Staphylococcus aureus in Los Angeles. N Engl J Med. 2005;352:1445-53.

6. Gillet $Y$, Issartel B, Vanhems P, Fournet JC, Lina G, Bes M, et al. Association between Staphylococcus aureus strains carrying gene for Panton-Valentine leukocidin and highly lethal necrotising pneumonia in young immunocompetent patients. Lancet. 2002;359:753-9.

7. Boyle-Vavra S, Ereshefsky B, Wang CC, Daum RS. Successful multiresistant community-associated methicillin-resistant Staphylococcus aureus lineage from Taipei, Taiwan, that carries either the novel Staphylococcal chromosome cassette mec (SCCMec) type VT or SCCmec type IV. J Clin Microbiol. 2005:43:4719-30

8. Robinson JO, Pearson JC, Christiansen KJ, Coombs GW, Murray RJ. Community-associated versus healthcare-associated methicillin-resistant Staphylococcus aureus bacteraemia: a 10-year retrospective review. Eur J Clin Microbiol Infect Dis. 2009:28:353-61.

9. National Health and Medical Research Council, Commonwealth of Australia: When does quality assurance in health care require independent ethical review? Advice to institutions, human research ethics committees and health care professionals 2003. [http://www.nhmrc.gov.au/guidelines/ publications/e46]

10. Clinical and Laboratory Standards Institute. Performance standards for antimicrobial testing: $18^{\text {th }}$ informational supplement. CLSI document M100-S18. Wayne, PA: Clinical and Laboratory Standards Institute; 2008.

11. Costa AM, Kay I, Palladino S. Rapid detection of mecA and nuc genes in Staphylococci by real-time multiplex polymerase chain reaction. Diagn Microbiol and Infect Dis. 2005;51:13-7.

12. O'Brien FG, Pearman JW, Gracey M, Riley TV, Grubb WB. Community strain of methicillin-resistant Staphylococcus aureus involved in a hospital outbreak. J Clin Microbiol. 1999;37:2858-62.

13. Fey PD, Said-Salim B, Rupp ME, Hinrichs SH, Boxrud DJ, Davis CC, et al. Comparative molecular analysis of community- or hospital-acquired methicillin-resistant Staphylococcus aureus. Antimicrob Agents Chemother. 2003;47:196-203.

14. Masiuk H, Kopron K, Grumann D, Goerke C, Kolata J, Jursa-Kulesza J, et al. Association of recurrent furunculosis with Panton-Valentine leukocidin and the genetic background of Staphylococcus aureus. J Clin Microbiol. 2010;48:1527-35.

15. Neela V, Ehsanollah GR, Zamberi S, Van Belkum A, Mariana NS. Prevalence of Panton-Valentine leukocidin genes among carriage and invasive Staphylococcus aureus isolates in Malaysia. Int J Infect Dis. 2009;13:e131-132.

16. Munckhof WJ, Nimmo GR, Schooneveldt JM, Schlebusch S, Stephens AJ Williams G, et al. Nasal carriage of Staphylococcus aureus, including community-associated methicillin-resistant strains, in Queensland adults. Clin Microbiol Infect. 2009;15:149-55.

17. Muttaiyah S, Coombs G, Pandey S, Reed P, Ritchie S, Lennon D, et al. Incidence, risk factors, and outcomes of Panton-Valentine leukocidin-positive methicillin-susceptible Staphylococcus aureus infections in Auckland, New Zealand. J Clin Microbiol. 2010:48:3470-4.

18. Tong SY, Lilliebridge RA, Bishop EJ, Cheng AC, Holt DC, McDonald MI, et al. Clinical correlates of Panton-Valentine leukocidin (PVL), PVL isoforms, and clonal complex in the Staphylococcus aureus population of Northern Australia. J Infect Dis. 2010:202:760-9.

19. Robinson DA, Kearns AM, Holmes A, Morrison D, Grundmann H, Edwards G, et al. Re-emergence of early pandemic Staphylococcus aureus as a community-acquired meticillin-resistant clone. Lancet. 2005;365:1256-8.

20. Donahue JA, Baldwin JN. Hemolysin and leukocidin production by $80 / 81$ strains of Staphylococcus aureus. J Infect Dis. 1996;116:324-8.

21. Gauduchon V, Cozon G, Vandenesch F, Genestier AL, Eyssade N, Peyrol S, et al. Neutralization of Staphylococcus aureus Panton Valentine leukocidin by intravenous immunoglobulin in vitro. J Infect Dis. 2004;189:346-53.

22. Nimmo GR, Schooneveldt JM, Sutherland JL, Power S, Olesen D, Selvey C, et al. Epidemiology of non-multiresistant methicillin-resistant Staphylococcus aureus infection in Queensland, Australia: associations with indigenous populations and Panton-Valentine leukocidin. Eur J Clin Microbiol Infect Dis. 2010;29:1253-9.

23. Lina G, Piemont $Y$, Godail-Gamot F, Bes M, Peter MO, Gauduchon $V$, et al. Involvement of Panton-Valentine leukocidin-producing Staphylococcus aureus in primary skin infections and pneumonia. Clin Infect Dis. 1999;29:1128-32.

24. Shallcross $\sqcup$, Fragaszy E, Johnson AM, Hayward AC. The role of Panton-Valentine leucocidin toxin in Staphylococcal disease: a systematic review and meta-analysis. Lancet Infect Dis. 2013;13:43-54.

25. Al-Rawahi GN, Reynolds S, Porter SD, Forrester L, Kishi L, Chong T, et al. Community-associated CMRSA-10 (USA-300) is the predominant strain among methicillin-resistant Staphylococcus aureus strains causing skin and soft tissue infections in patients presenting to the emergency department of a Canadian tertiary care hospital. J Emerg Med. 2010;38:6-11.

26. Murray RJ, Robinson JO, White JN, Hughes F, Coombs GW, Pearson JC, et al. Community-acquired pneumonia due to pandemic A (H1N1) 2009 influenza virus and methicillin resistant Staphylococcus aureus co-infection. PloS One. 2010;5:e8705.

27. Gillet $Y$, Dumitrescu $O$, Tristan A, Dauwalder O, Javouhey E, Floret $D$, et al. Pragmatic management of Panton-Valentine leukocidin-associated staphylococcal diseases. Int J Antimicrob Agents. 2010;38:457-64.

\section{Submit your next manuscript to BioMed Central and take full advantage of:}

- Convenient online submission

- Thorough peer review

- No space constraints or color figure charges

- Immediate publication on acceptance

- Inclusion in PubMed, CAS, Scopus and Google Scholar

- Research which is freely available for redistribution 